\title{
ТРАНСАБДОМИНАЛЬНОЕ УЛЬТРАЗВУКОВОЕ СКАНИРОВАНИЕ КАК СКРИНИНГОВЫЙ ЭТАП ДИАГНОСТИКИ ТУБЕРКУЛЕЗНОГО ПЕРИТОНИТА
}

Д. В. Плоткин ${ }^{1,2}$, О. В. Кириллова ${ }^{1}$, А. В. Никаноров ${ }^{3}$, М. Н. Решетников ${ }^{1}$, А. О. Штыхно ${ }^{1}$, Е. О. Лошкарева², Е. С. Короткова², М. В. Синицын

${ }^{1}$ Московский городской научно-практический центр борьбы с туберкулезом, Москва, Россия

2 Российский национальный исследовательский медицинский университет имени Н. И. Пирогова, Москва, Россия

${ }^{3}$ Московский клинический научный центр имени А. С. Логинова, Москва, Россия

За последние годы отмечен рост числа случаев туберкулезного перитонита. Туберкулез брюшины - сложный объект для диагностики и нередко верификация диагноза происходит со значительными задержками. В клинической практике предложен непродолжительный и доступный метод лучевой диагностики при подозрении на туберкулезный перитонит - ультразвуковое исследование (УзИ). Целью работы было описать эхо-семиотику туберкулезного перитонита с созданием интегральной шкалы оценки значимости отдельных эхо-симптомов туберкулеза брюшины и определить роль УЗ-сканирования в верификации диагноза. Произведен ретроспективный анализ инвазивной и УЗ-диагностики 37 пациентов с подтвержденным туберкулезным перитонитом в период с 2009 по 2019 г. В качестве группы сравнения использовали такие же данные исследований у 28 больных с заболеваниями, часто имитирующими туберкулезный перитонит (канцероматозом и саркоидозом брюшины, неспецифическим асцитом). Выделень прямые и косвенные признаки поражения брюшины при туберкулезе, на основании этого создана интегральная шкала оценки значимости отдельных эхо-симптомов. С этой целью каждому эхо-симптому присваивали от 0 до 3 баллов. При оценке визуализации описанных эхо-признаков возможно прогнозировать вероятность туберкулезной этиологии заболевания. Получены следующие статистические данные: низкая вероятность наличия туберкулезного перитонита при оценке визуализации всех описанных эхо-симптомов - до 4 баллов, средняя - от 5 до 8 баллов, высокая 9 и более баллов. Разработанная интегральная схема оценки УЗ-признаков позволяет клиницисту на основании эхограсического исследования верифицировать туберкулезный перитонит или выбрать дальнейшую тактику в диагностическом поиске.

Ключевые слова: ультразвуковое исследование, туберкулез брюшины, перитонит, канцероматоз, саркоидоз

Вклад авторов: Д. В. Плоткин, М. Н. Решетников, А. В. Никаноров, М. В. Синицын - разработка концепции и дизайна исследования, общая ответственность; О. В. Кириллова, А. О. Штыхно, Е. О. Лошкарева - сбор материала; Е. С. Короткова, Д. В. Плоткин - статистическая обработка данных; Д. В. Плоткин, М. Н. Решетников, О. В. Кириллова, А. В. Никаноров - анализ полученных данных; Д. В. Плоткин, М. Н. Решетников подготовка текста; О. В. Кириллова, А. О. Штыхно, А. В. Никаноров - редактирование.

Соблюдение этических стандартов: исследование одобрено этическим комитетом Московского научно-практического центра борьбы с туберкулезом (протокол № 12 от 9 декабря 2019 г.). Все пациенты подписали добровольное информированное согласие.

$\triangle$ Для корреспонденции: Дмитрий Владимирович Плоткин ул. Островитянова, д. 1, г. Москва, 117997; kn13@list.ru

Статья получена: 17.03.2020 Статья принята к печати: 04.04.2020 Опубликована онлайн: 12.04.2020

DOI: $10.24075 /$ vrgmu.2020.018

\section{TRANSABDOMINAL ULTRASOUND AS A SCREENING STAGE FOR THE DIAGNOSIS OF TUBERCULOUS PERITONITIS}

\author{
Plotkin DV ${ }^{1,2}$, Kirillova OV ${ }^{1}$, Nikanorov AV ${ }^{3}$, Reshetnikov MN¹, Shtykhno AO ${ }^{1}$, Loshkareva EO², Korotkova ES², Sinitsyn MV ${ }^{1}$ \\ ${ }^{1}$ Moscow Research and Clinical Center for TB Control, Moscow, Russia \\ 2 Pirogov Russian National Research Medical University, Moscow, Russia \\ ${ }^{3}$ Loginov Moscow Clinical Scientific Practical Center, Moscow, Russia
}

In recent years, the incidence of tuberculous peritonitis increased. Peritoneal tuberculosis is difficult to diagnose, and often the diagnosis is verified with significant delay. In clinical practice, a quick and affordable diagnostic radiology method, ultrasonography (USG), is proposed for patients with suspected tuberculous peritonitis. The study was aimed to describe the sonographic semiology of tuberculous peritonitis, to create the integrated scale for the individual peritoneal tuberculosis sonographic symptoms significance assessment, and to determine the role of ultrasound imaging in the diagnosis verification. Retrospective study of the invasive and ultrasound investigation results of 37 patients with confirmed tuberculous peritonitis was carried out in 2009-2019. Similar data obtained by investigation of 28 patients with the disorders which often mimic the tuberculous peritonitis (peritoneal carcinomatosis and sarcoidosis, non-specific ascites) were used as a comparison group. Direct and indirect signs of peritoneal lesion in patients with tuberculosis were identified. On the basis of that, an integral scale for the individual sonographic symptoms significance assessment was created. Each sonographic symptom received a 0-3 score. Assessment of those sonographic signs visualization allowed us to evaluate the probability of the disorder's tuberculous etiology. The following data were obtained: score under 4 corresponded to low probability, score 5-8 corresponded to medium probability, and score over 9 corresponded to high probability of tuberculous peritonitis based on the visualization of all described songraphic symptoms. The proposed integrated scale for the sonographic signs assessment allows the clinician to verify the tuberculous peritonitis diagnosis based on the ultrasound imaging data or to select the further tactics of diagnosis.

Keywords: ultrasonography, peritoneal tuberculosis, peritonitis, carcinomatosis, sarcoidosis

Author contribution: Plotkin DV, Reshetnikov MN, Nikanorov AV, Sinitsyn MV — study concept and design, overall management; Kirillova OV, Shtykhno AO, Loshkareva EO — sample collection; Korotkova ES, Plotkin DV — statistical analysis; Plotkin DV, Reshetnikov MN, Kirillova OV, Nikanorov AV — data analysis; Plotkin DV, Reshetnikov MN — manuscript writing; Kirillova OV, Shtykhno AO, Nikanorov AV — editing.

Compliance with ethical standards: the study was approved by the Ethics Committee of the Moscow Research and Clinical Center for TB Control (protocol №.12 dated December 9, 2019). The informed consent was submitted by all study participants.

$\triangle$ Correspondence should be addressed: Dmitry V. Plotkin Ostrovityanova, 1, Moscow, 117997; kn13@list.ru

Received: 17.03.2020 Accepted: 04.04.2020 Published online: 12.04 .2020

DOI: 10.24075/brsmu.2020.018 
Туберкулезный перитонит вновь появился в европейских и российских клиниках после 45 лет забвенья. Этому способствовали пандемия ВИЧ-инфекции, миграция населения из эндемичных регионов, появление штаммов микобактерий с широкой лекарственной устойчивостью и медикаментозные иммуносупрессии. Туберкулез брюшины (первичный перитонит) является хроническим воспалительным процессом с неспецифическими клиническими проявлениями, что нередко вызывает значительные трудности и задержку в диагностике [1-3]. По мнению большинства исследователей, наиболее точным методом верификации туберкулеза брюшины служит лапароскопия с биопсией пораженных участков серозного покрова брюшной полости [4-5]. Рост микобактерий туберкулеза (МБТ) из экссудата показывает положительный результат на 4-6-й неделе после посева только в 10\% случаев, а ПЦР-диагностика выпота информативна лишь в трети наблюдений и также требует проведения инвазивного вмешательства [5-6]. В клинической практике предложень методы непродолжительной по времени и доступной лучевой диагностики при подозрении на туберкулезный перитонит - ультразвуковое исследование (УЗИ) и компьютерная томография [1, 7, 8], однако в большинстве случаев они не позволяют точно идентифицировать воспалительный процесс на брюшине.

Ультрасонография - наиболее безопасный, эффективный и экономичный способ визуализации, способный помочь клиницистам принять решения по диагностике и своевременному лечению туберкулезного перитонита. Сегодня трансабдоминальная сонография играет важную роль в оценке воспалительных, доброкачественных и злокачественных заболеваний брюшины, как в амбулаторных, так и в стационарных условиях. В медицинской литературе описаны различные эхо-признаки, позволяющие предположить туберкулезный перитонит, а также способы, позволяющие диффееренцировать его с канцероматозом брюшины и неспецифическим асцитом [7-11]. Точность этих методик напрямую зависит от квалификации и опыта врача, а также класса используемой аппаратуры. Последние 10 лет в большинстве исследований приводятся примеры отдельных наблюдений из практики по визуализации перитонеальных изменений при туберкулезе, однако полностью отсутствует анализ встречаемости признаков и их сочетаний $[9,11]$.

Цель данной работы - описать эхо-семиотику туберкулезного перитонита с созданием интегральной шкалы оценки значимости отдельных эхо-симптомов туберкулеза брюшины и определить роль ультразвукового (УЗ) сканирования в верификации диагноза.

\section{ПАЦИЕНТЫ И МЕТОДЫ}

В период с 2009 по 2019 г. В туберкулезном хирургическом отделении клиники № 2 Московского городского научно-практического центра борьбы с туберкулезом г. Москвы с целью определения основных эхо-симптомов туберкулезного перитонита было проведено ретроспективное исследование результатов УЗ-диагностики 37 пациентов с туберкулезным перитонитом, у которых ведущим клиническим синдромом было наличие большого количества выпота в брюшной полости (экссудативная, адгезивно-слипчивая, казеозная и смешанная формы туберкулезного перитонита). Диагноз туберкулеза брюшины был установлен у всех 37 больных при гистологическом (100\%) и бактериоскопическом $(81,1 \%)$ исследованиях биоптатов брюшины, полученных с помощью лапароскопических инвазивных методик, что и являлось критерием включения пациентов в исследование. Среди госпитализированных больных преобладали лица мужского пола (мужчины - 22; 59,5\%, женщины 15; 40,5\%) в возрасте 20-65 лет (медиана - 37,2 года). ВИЧ-позитивный статус был зарегистрирован у 23 больных (62,7\%), а туберкулез легких - у 34 пациентов (91,6\%) с превалированием инфильтративных и диссеминированных форм. Критерий исключения пациентов из исследования: отсутствие гистологической верификации туберкулеза брюшины.

Для группы сравнения был произведен отбор пациентов, у которых при лапароскопическом, гистологическом и лабораторном исследованиях верифицированы заболевания, наиболее часто имитирующие туберкулезный перитонит, как в клинической картине, так и при УЗИ. В контрольную группу были включены 28 пациентов с асцитом различной неспецифической этиологии (21 пациент; 75,0\%), саркоидозом брюшины (1 пациент; 3,5\%) и канцероматозом брюшины (6 пациентов; 21,5\%). Возраст пациентов варьировал от 29 до 54 лет (медиана 36,1 года); туберкулез легких был диагностирован у всех пациентов, а ВИЧ-позитивный статус имел место у 10 (35,7\%) больных контрольной группы. Критерии исключения пациентов из контрольной группы: наличие туберкулезных гранулем и КУМ в биоптатах брюшины; положительный результат ПЦР-диагностики выпота на ДНК МБТ.

В комплекс обследования пациентов включали полипозиционную рентгенографию и рентгеновскую компьютерную томографию грудной клетки, комплексное УЗи органов брюшной полости, диагностическую видеолапароскопию, лабораторные и морфологические методы исследования. Диагностические исследования расширяли применением гистологического, цитологического, и молекулярно-генетического анализов интраоперационного материала (биоптатов и экссудата). При проведении микробиологических исследований использовали бактериоскопию по Цилю-Нильсену для обнаружения кислотоустойчивых микобактерий (КУМ), посевы на плотные и жидкие питательные среды с применением автоматизированных систем.

УЗИ органов брюшной полости выполняли на многофункциональном (экспертного класса) аппарате Toshiba Aplio 500 (Toshiba; Япония) и портативном аппарате LOGIC ER7 (General Electric; Южная Корея), работающих в режиме серой шкалы и реального времени. Исследование брюшной полости проводили конвексным датчиком 2,5-5,0 МГц с оценкой наличия и распространенности свободной жидкости, состояния селезенки, печени. Для оценки состояния петель кишечника, брыжейки, большого и малого сальника и лимфатического аппарата использовали высокочастотный линейный датчик 10-15 МГц.

Оперативные вмешательства (лапароскопия с биопсией или лапаротомия) были выполнены в плановом порядке при наличии асцита неясной этиологии или с целью уточнения характера патологического процесса, выявленного при УЗИ и компьютерной томографии, в экстренном порядке при клинической картине, не позволяющей исключить перитонит.

Критерием верификации диагноза служило обнаружение в биоптатах брюшины туберкулезных гранулем, КУМ при бактериоскопическом исследовании и положительный результат ПЦР-диагностики выпота, а также сочетание этих признаков. 
Статистическую обработку результатов исследования проводили с использованием стандартных статистических методов и пакета программ Statgraphics Centurion 18 (Statgraphics Technologies, Inc.; США), языка Python 3.6 (Python Software Foundation; США) и теоремы Т. Байесса [12].

\section{РЕЗУЛЬТАТЫ ИССЛЕДОВАНИЯ}

Для оценки эхо-симптомов туберкулезного перитонита нами были выделены следующие прямые признаки, относящиеся непосредственно к воспалительному процессу в серозных листках и связанной с ним экссудацией: наличие свободной жидкости и ее расположение в брюшной полости, однородность экссудата, наличие фибриновых септперегородок или полуперегородок в брюшной полости, слоистость и неоднородность брюшины и сальника, наличие диссеминатов на серозных листах.

K косвенным признакам туберкулезного поражения брюшины мы отнесли изменения со стороны петель кишечника, увеличение и неоднородность селезенки, возможность визуализации сгруппированных увеличенных мезентериальных лимфатических узлов. Косвенные признаки напрямую не отражают воспалительный процесс в листках брюшины, но могут быть следствием длительно существующего асцита (утолщение стенок кишечника), а также реактивного или специфического лимфаденита и спленита при абдоминальном туберкулезе.

При выполнении УЗ-сканирования брюшной полости у лиц с туберкулезным перитонитом во всех случаях была выявлена свободная жидкость различного объема. Выпот во всех отделах брюшной полости удалось визуализировать у 19 пациента (51,4\%). В восьми наблюдениях $(21,6 \%)$ свободная жидкость была локализована преимущественно в малом тазу и между петлями тонкой кишки, а у 10 пациентов $(27,0 \%)$ выявлено осумкованное расположение свободной жидкости в брюшной полости с формированием полусепт или полных перегородок из пластов фибрина. Неоднородность выпота за счет свободно флотирующих пластов фрибрина и мелких фрибриновых секвестров диаметром до 6-8 мм была отмечена в подавляющем большинстве случаев (75,7\%). Слоистость и гетерогенность листков париетальной брюшины на отдельных участках, преимущественно в проекции илеоцекальной области, отмечены в 14 случаях (37,8\%), причем в одном наблюдении в толще передней брюшной стенки были визуализированы очаги казеозной деструкции (рис. 1). Неоднородность и слоистость большого сальника обнаружены у 9 пациентов (24,3\%). Характерные для туберкулезного перитонита бугорковые диссеминаты визуализированы у 9 больных (24,3\%), их размеры колебались в пределах 5-9 мм и были описаны как возвышающиеся над париетальной брюшиной гиперэхогенные аваскулярные очаговые образования с неровным контуром (рис. 1, 2).

Другие УЗ-признаки, позволяющие предположить туберкулезную природу изменений на брюшине, имели косвенный характер и включали в себя эхо-симптомы вовлечения в патологический процесс висцеральной брюшины кишечника, селезенки и мезентериального лимфатического аппарата. Изменения со стороны стенок кишки имели место у 7 (18,9\%) пациентов, как правило, отмечалось локальное утолщение кишечной стенки свыше 3 мм на протяжении не более 45-60 мм. В 6 случаях (16,2\%) были выявлены расширение просвета кишечника более 3335 мм и ослабление или полное отсутствие перистальтики, что трактовалось нами как парез. Увеличение брыжеечных лимфатических узлов более 10-16 мм в диаметре с неоднородной структурой зарегистрировано у 19 пациентов (51,4\%). Спленомегалия с неоднородностью структуры селезенки была отмечена у 5 больных (13,5\%). Сравнительные результаты УЗ-сканирования пациентов контрольной группы представлены в табл. 1.

Для создания интегральной шкалы оценки вероятности диагностики туберкулезного перитонита при Узи были рассмотрены все варианты сочетания признаков (эхо-симптомов) между собой. С этой целью каждому эхо-симптому в зависимости от его значимости и специфичности было присвоено от 0 до 3 баллов. Первые три признака (распределение свободной жидкости) определяют первое полное вероятностное пространство события (наличие выпота в брюшной полости). Бугорковые диссеминаты различных размеров можно считать вторым полным вероятностным пространством

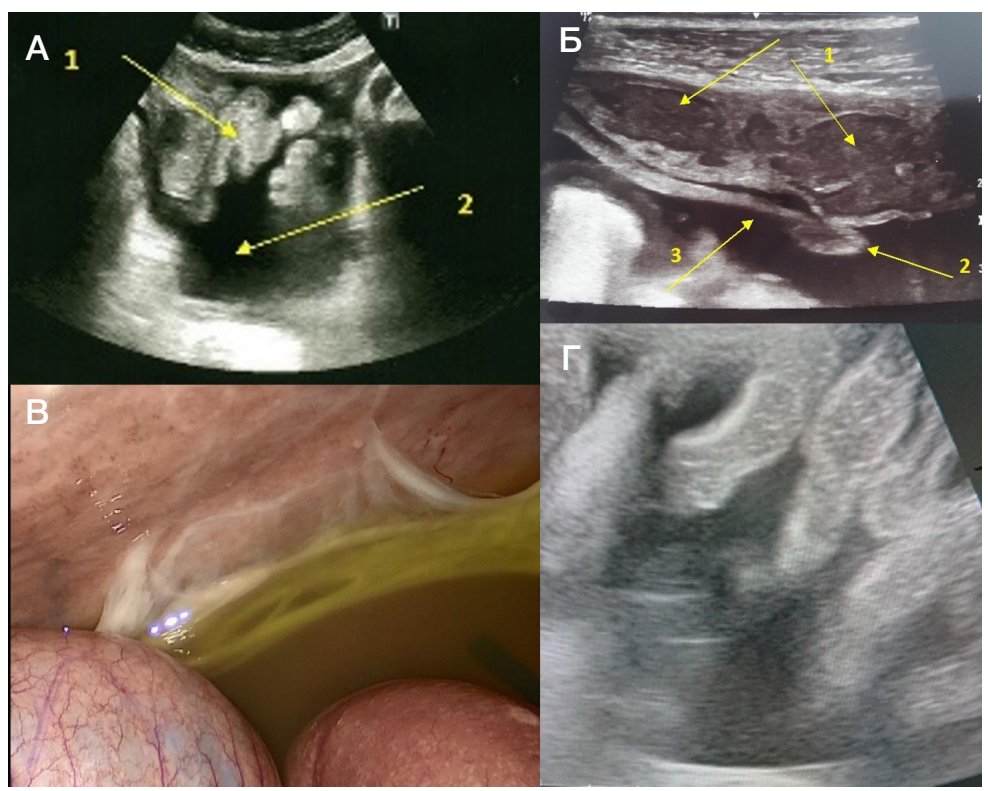

Рис. 1. Туберкулезный перитонит. А. Эхограмма: 1 - паретичные петли кишки, 2 - экссудат. Б. Эхограмма: 1 - казеозный абсцесс в толще брюшины, 2 - туберкулезный диссеминат, 3 - слоистость париетальной брюшины. В. Лапароскопия. Г. Эхограмма: неоднородный фибринозный выпот и паретичные петли тонкой кишки 


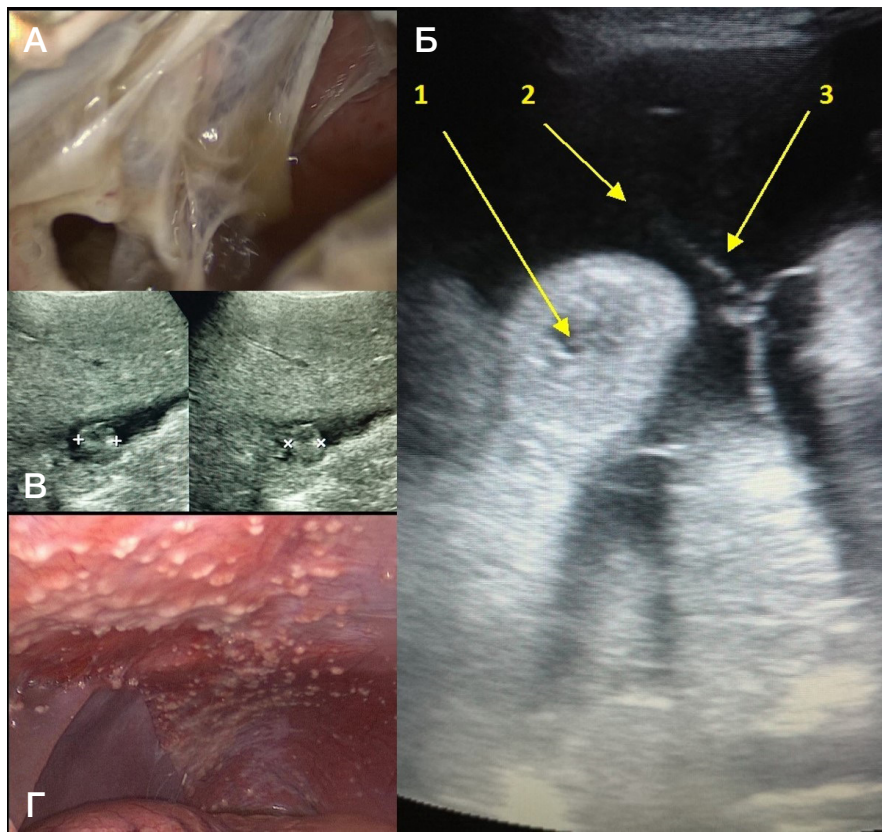

Рис. 2. Туберкулезный перитонит. А. Лапароскопия: фибриновые наложения, образующие перегородки. Б. Эхограмма: 1 - петли кишки, 2 - экссудат, 3 - фибриновые тяжи, образующие перегородки. В. Эхограмма: туберкулезный диссеминат (7 мм). Г. Лапароскопия: туберкулезные диссеминаты на париетальной брюшине

события (по признаку размера диссеминатов). Остальные же признаки (неоднородность выпота с фрагментами фибрина, слоистость и неоднородность листков париетальной брюшины и сальника, казеозные абсцессы в толще брюшины) независимы между собой. Далее были произведены расчеты всех вариантов сочетания признаков между собой на языке Python 3.6 с использованием теоремы Т. Байесса, которая позволяет найти данную вероятность исходя из представленных данных. Предположения о приближении биноминальных распределений к нормальным и вывод о вероятностном отнесении больных по группам (низкая, средняя, высокая) выполнены с использованием библиотек stats и numpy (табл. 2).

\section{ОБСУЖДЕНИЕ РЕЗУЛЬТАТОВ}

Таким образом, можно отметить, что визуальные признаки туберкулезного перитонита, доброкачественных гранулематозов брюшины (саркоидоза), неспецифического асцита и перитонеального канцероматоза во многом схожи и в большинстве случаев ставят исследователя перед диагностическим выбором.

Как показывает собственный опыт проведения УЗ-диагностики при туберкулезном перитоните и данные литературы [7-10], основным эхо-симптомом служит неоднородная свободная или осумкованная жидкость в брюшной полости (75-80\%). Характерным признаком, отличающим туберкулезный перитонит от неспецифического асцита, служит более частая (около 75\%) визуализация свободно располагающихся в экссудате секвестров фибрина, которые в отдельных случаях образуют полные или неполные перегородки, что ведет к осумковыванию выпота (рис. 2). Тем не менее подобные УЗ-находки в 50\% случаев могут встречаться и при канцероматозе брюшины [13]. В свою очередь для канцероматоза высока вероятность локации опухолевых

Таблица 1. Прямые и косвенные эхо-признаки туберкулезного перитонита. Заданный уровень значимости признака равен 0,95

\begin{tabular}{|l|c|c|c|}
\hline \multicolumn{1}{|c|}{ Эхо-симптом } & $\begin{array}{c}\text { Tуберкулезный перитонит } \\
n=37\end{array}$ & $\begin{array}{c}\text { Асцит и канцероматоз } \\
n=28\end{array}$ & Характер признака \\
\hline Свободная жидкость во всех отделах брюшной полости & $51,4 \% \pm 16,1$ & $82,1 \% \pm 14,1$ \\
\hline Свободная жидкость в малом тазу и между петлями кишечника & $21,6 \% \pm 13,2$ & $3,6 \% \pm 6,8$ \\
\hline Осумкованная жидкость & $27,0 \% \pm 14,3$ & $14,3 \% \pm 12,9$ \\
\hline Неоднородность выпота & $75,7 \% \pm 13,8$ & $3,6 \% \pm 6,8$ \\
\hline $\begin{array}{l}\text { Слоистость и неоднородность листков париетальной } \\
\text { брюшины и сальника }\end{array}$ & $37,8 \% \pm 15,6$ & 0 \\
\hline Казеозные абсцессы в толще брюшины & $2,7 \% \pm 1,6$ & 0 \\
\hline Бугорковые диссеминаты брюшины & $24,3 \% \pm 13,8$ & $10,7 \% \pm 11,5$ \\
\hline Утолщение стенки кишечника & $18,9 \% \pm 12,6$ & $21,4 \% \pm 15,2$ \\
\hline Парез кишечника & $16,2 \% \pm 11,9$ & $10,7 \% \pm 11,5$ \\
\hline Спленомегалия & $13,5 \% \pm 11,0$ & $71,4 \% \pm 16,7$ \\
\hline $\begin{array}{l}\text { Увеличение и неоднородность мезентериальных } \\
\text { лимфатических узлов }\end{array}$ & $51,4 \% \pm 16,1$ & $14,3 \% \pm 12,9$ \\
\hline Увеличение печени и обеднение сосудистого рисунка & $27,0 \% \pm 14,3$ & $82,1 \% \pm 14,2$ \\
\hline Расширение воротной вены и диффузные изменения печени & $8,1 \% \pm 8,8$ & $85,7 \% \pm 12,9$ \\
\hline
\end{tabular}




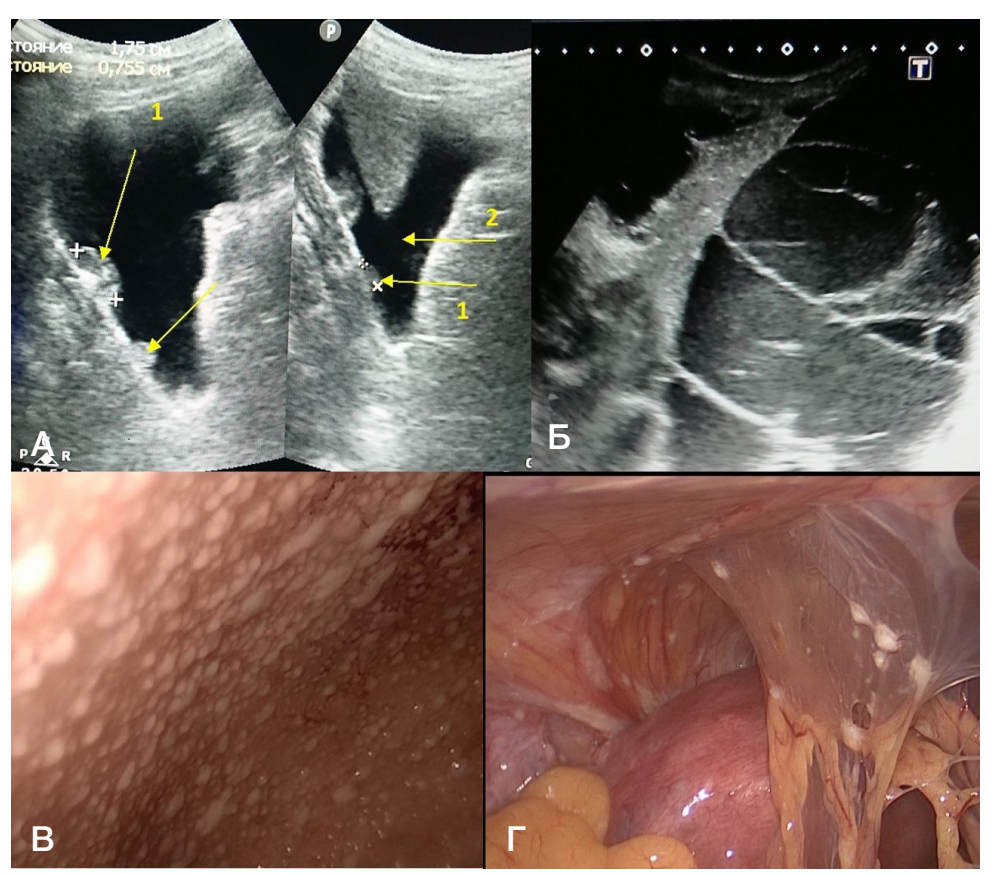

Рис. 3. Гранулематозные перитониты и канцероматоз. А. Эхограмма: канцероматоз брюшины при раке сигмовидной кишки. 1 - опухолевые диссеминаты (17 и 8 мм), 2 - асцит. Б. Эхограмма: осумкованный асцит при раке яичника. В. Лапароскопия: канцероматоз париетальной брюшины. Г. Лапароскопия: саркоидоз брюшины

диссеминатов диаметром 5-18 мм (рис. 3). По данным нашего исследования, подобные гиперэхогенные образования на брюшине можно обнаружить и при доброкачественных гранулематозах, например при саркоидозе (рис. 3), что тоже не дает основания выделить этот признак в специфический. Туберкулезная диссеминация на брюшине, как правило, имеет меньший диаметр и максимальный размер бугорка до 9 мм (медиана 4-6 мм) [11, 13-16]. Более достоверным эхо-симптомом туберкулезного перитонита является неоднородность (слоистость) участков париетальной брюшины и большого сальника, однако этот признак удается определить чуть более чем в трети случаев. Наиболее распространенной находкой является протяженное гипоэхогенное утолщение серозного париетального листка от 4 до 8 мм, что отражает хронический характер воспаления. Такой вариант визуализации утолщенной брюшины, по данным различных исследователей, все же с высокой долей вероятности соответствует туберкулезному процессу $[8,17-20]$ и реже встречается при перитонеальном карциноматозе, где утолщение серозного покрова чаще всего непротяженное и узловатое [20]. Вовлечение большого сальника и его изменения разные авторы описывают как характерный признак туберкулезного оментита или канцероматоза примерно с одинаковой частотой, поэтому мы считаем этот признак специфичным для обоих заболеваний, учитывая, что визуализировать сальниковые изменения удается достаточно редко (менее 25\% наблюдений) [21].

Косвенные признаки поражения брюшины при туберкулезе, такие как лимфаденопатия с аденомегалией, встречаются у половины больных туберкулезным перитонитом, что отражает патогенетический характер лимфогенной диссеминации в листки брюшины. Изменения в мезентериальном лимфатическом аппарате чаще всего носят деструктивный характер, и лимфатические узлы визуализируются как сгруппированные анэхогенные округлые структуры диаметром более 10 мм. По данным литературы, частота содружественного поражения внутрибрюшных лимфатических узлов и брюшины составляет 10-54\% [22-24].

Таким образом, понятно, что туберкулезный перитонит не имеет каких-либо эталонных или уникальных эхо-

Таблица 2. Интегральная таблица встречаемости эхо-симптомов при туберкулезном перитоните

\begin{tabular}{|l|c|}
\hline \multicolumn{1}{|c|}{ Эхо-признак } & Баллы \\
\hline Свободная жидкость во всех отделах брюшной полости & 0 \\
\hline Свободная жидкость в малом тазу и между петлями кишечника & 2 \\
\hline Осумкованная жидкость в брюшной полости с перегородками & 2 \\
\hline Неоднородность выпота с фрагментами фибрина & 3 \\
\hline Слоистость и неоднородность листков париетальной брюшины и сальника & 3 \\
\hline Казеозные абсцессы в толще брюшины & 3 \\
\hline Бугорковые диссеминаты брюшины до 9 мм в диаметре & 0 \\
\hline Бугорковые диссеминаты брюшины более 9 мм в диаметре & 1 \\
\hline Косвенные признаки: изменения стенки кишки и мезентериальная лимфаденопатия \\
\hline Вероятность наличия туберкулезного перитонита \\
\hline высокая & средняя \\
\hline$\geq 9$ баллов & 5-8 баллов \\
\hline
\end{tabular}


симптомов, что крайне затрудняет его диагностику при УЗИ. В то же время различные варианты сочетания некоторых прямых и косвенных признаков повышают вероятность правильной трактовки УЗ-картины при туберкулезе брюшины.

На основании расчетов и распределения вероятностей получены следующие статистические данные: низкая вероятность наличия туберкулезного перитонита при оценке визуализации всех описанных эхо-симптомов до 4 баллов, средняя - 5-8 баллов, высокая - 9 и более баллов. Основываясь на сумме полученных баллов в результате УЗ-сканирования, можно предложить диагностический алгоритм, в котором четко указаны дальнейшие диагностические и лечебные стратегии для каждого из вариантов сочетания эхо-симптомов и наличия у пациента ВИЧ-инфекции и/или туберкулеза легких (рис. 4).

\section{ВЫВОДЫ}

Полученная интегральная схема для оценки УЗ-признаков достаточно проста и позволяет клиницисту на основании результатов эхографического исследования выбрать дальнейшую тактику в диагностическом поиске и своевременно начать лечение. Предложенная схема может также войти в алгоритм диагностического поиска при любой форме асцита или гранулематозного перитонита.

Диагностика туберкулеза брюшины по-прежнему остается сложной задачей, ввиду неспецифичности клинической симптоматики и подострого характера течения. Доступное и распространенное в медицинской практике трансабдоминальное ультразвуковое исследование может стать скрининговым диагностическим исследованием первой линии для верификации туберкулезного перитонита. Туберкулез брюшины следует принимать во

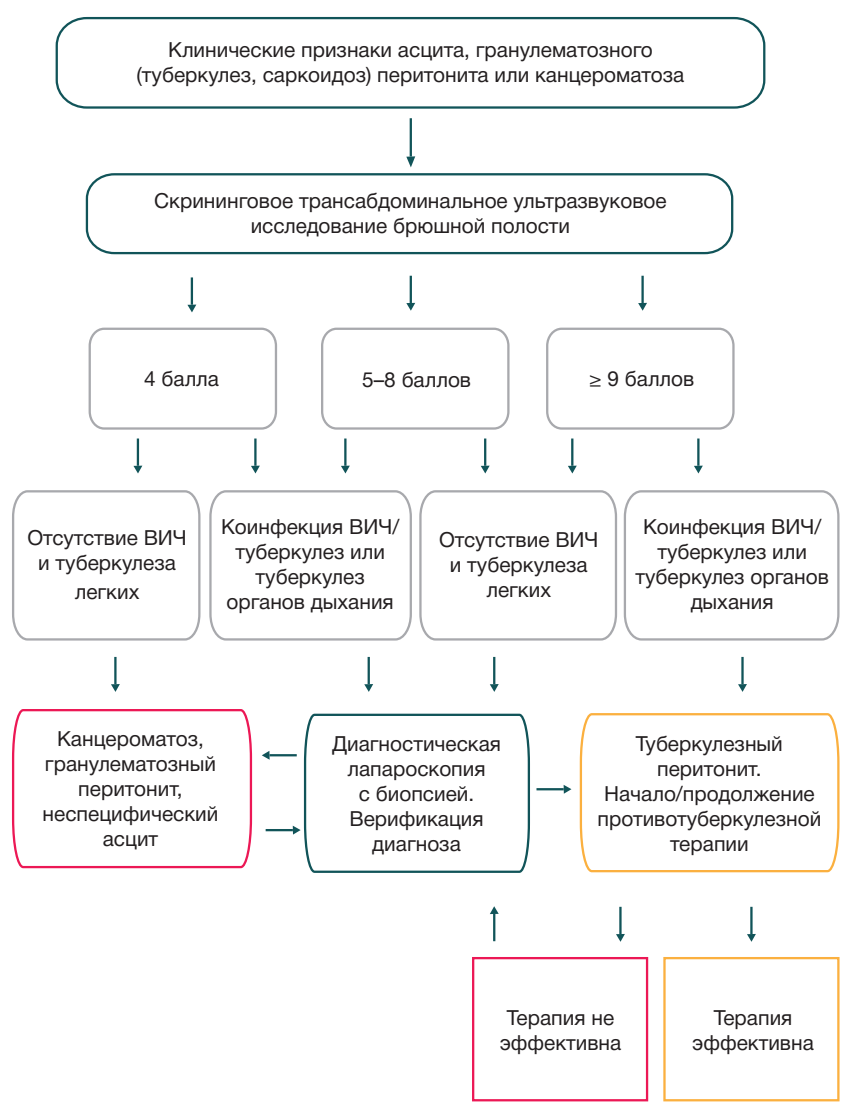

Рис. 4. Тактический алгоритм диагностики туберкулезного перитонита

внимание во всех случаях дифференциальной диагностики у пациентов с неясным асцитом, туберкулезом легких и иммуносупрессией.

\section{Литература}

1. Gupta P, Kumar S, Sharma V, Mandavdhare H, Dhaka N, Sinha SK et al. Common and uncommon imaging features of abdomina tuberculosis. Journal of Medical Imaging and Radiation Oncology. 2019; 63 (3): 329-39. DOI: 10.1111/1754-9485.12874.

2. Синицын М. В., Белиловский Е. М., Соколина И. А Решетников М. Н., Титюхина М. В., Батурин О. В. Внелегочные локализации туберкулеза у больных ВИЧ-инфекцией. Туберкулез и болезни легких. 2017; 95 (11): 19-25. DOI: 10.21292/2075-1230-2017-95-11-19-25.

3. Ködmön C, Zucs P, Van der Werf MJ. Migration-related tuberculosis: Epidemiology and characteristics of tuberculosis cases originating outside the European Union and European Economic Area, 2007 to 2013. Eurosurveillance. 2016; (21): 2327. DOI: 10.2807/1560-7917.ES.2016.21.12.30164.

4. Chow KM, Chow VC, Szeto CC. Indication for peritoneal biopsy in tuberculous peritonitis. The American Journal of Surgery. 2003; (185): 567-73. DOI: 10.1016/s0002-9610(03)00079-5.

5. Плоткин Д. В., Синицын М. В., Решетников М. Н., Харитонов С. В., Скопин М. С., Соколина И. А. Туберкулезный перитонит. «Забытая» болезнь. Хирургия. Журнал им. Н. И. Пирогова. 2018; (12): 38-44. DOI: 10.17116/hirurgia201812138.

6. Vaid U, Kane GC. Tuberculous Peritonitis. Microbiology Spectrum. 2017; (5): 324-29. DOI: 10.1128/microbiolspec.TNMI7-0006-2016.

7. Demirkazik FB, Akhan O, Ozmen MN, Akata D. US and CT findings in the diagnosis of tuberculous peritonitis. Acta Radiologica. 1996; 37 (4): 517-20. DOI: 10.1177/02841851960373P217.

8. Portielje JE, van der Werf SD, Mutsaers JA, Lohle PN, Puylaert JB. Peritonitis tuberculosa echografisch te herkennen [Echographic recognition of tuberculous peritonitis]. Nederlands Tijdschrift voor

Geneeskunde. 1997; 141 (2): 89-93.

9. Мойдунова Н. К., Турдумамбетова Г. К. Ультразвуковая картина абдоминального туберкулеза. Международный журнал прикладных и фундаментальных исследований. 2017; (6): 111-3.

10. Востров А. Н., Митина Л. А., Казакевич В. И., Каприн А. Д., Степанов С. О., Гуц О. В. и др. Возможности ультразвуковой диагностики в дифференциации поражения брюшины при раке яичников и туберкулезе. Ультразвуковая и функциональная диагностика. 2017; (3): 60-71.

11. Husain A, Firdaus $H$, Panday P. Study of Comparison of high resolution sonography and computed tomography in evaluation of abdominal tuberculosis among patients in Lucknow, Uttar Pradesh, India. International Journal of Surgery. 2018; (5): 17139. DOI: 10.18203/2349-2902.isj20181388.

12. Goodman SN. Toward Evidence-Based Medical Statistics. 2: The Bayes Factor. Annals of Internal Medicine. 1999; (130): 1005-13.

13. Smereczyński A, Kołaczyk K, Bernatowicz E. Difficulties in differentiating the nature of ascites based on ultrasound imaging. Journal of Ultrasonography. 2017; 17 (69): 96-100. DOI: 10.15557/JoU.2017.0013.

14. Atzori S, Vidili G, Delitala G. Usefulness of ultrasound in the diagnosis of peritoneal tuberculosis. The Journal of Infection in Developing Countries. 2012; 6 (12): 886-90. DOI: 10.3855/ jidc.2654.

15. Weill FS, Costaz R, Guetarni S, Maltoni I, Rohmer P. Echographic diagnosis of peritoneal metastases in patients with ascites. European Journal of Radiology. 1990; (71): 365-8.

16. Степанов С. О., Митина Л. А., Гуц О. В., Беспалов П. Д. 
Визуализация перитонеальной диссеминации при ультразвуковом исследовании. Ультразвуковая и функциональная диагностика. 2013; 3 (4): 66-70.

17. N'dri K, Gbazi GC, Konan A, Kouadio, Koffi, N'dri N, et al. Apport de l'échographie dans le diagnostic de la tuberculose péritonéale ascitique. Médecine d'Afrique Noire. 1993; (40): 503-6.

18. Dafiri R, Imani F. Tuberculose abdominale. Encycl Méd Chir (Editions Scientifiques et Médicales Elsevier SAS, Paris, tous droits réservés). Radiodiagnostic - Appareil digestif 2001; 33010-A-30: 12.

19. Gastli H, Hassine W, Absesselem K, Gharbi HA. Echographic aspects of peritoneal tuberculosis. Apropos of 14 cases. European Journal of Radiology. 1983; (64): 325-9.

20. Mbengue A, Ndiaye AR, Amar N, Diallo M, et al. Ultrasonography of peritoneal tuberculosis. Journal of Ultrasonography. 2019; (19): 98-104. DOI: 10.15557/JoU.2019.0014.

21. BatraA, Gulati MS, Sarma D, Paul SB. Sonographic appearances in abdominal tuberculosis. Journal of Clinical Ultrasound. 2000; (28): 233-45. DOI: 10.1002/(sici)1097-0096(200006)28:5<233::aidjcu5>3.0.co;2-c.

22. Fall F, Ndiaye AR, Ndiaye B, Gning SB, Diop Y, Fall B. Peritoneal tuberculosis: a retrospective study of 61 cases at Principal hospital in Dakar. Journal of Gastroenterology and Hepatology. 2010; (4): 38-43.

23. Darré T, Tchaou M, Sonhaye L, Patassi AA, Kanassoua K, Tchangai B. Analyse d'une série de 44 cas de tuberculose péritonéale diagnostiqués au laboratoire d'anatomie pathologique du $\mathrm{CHU}$ Tokoin de Lomé (1993-2014). Bulletin de la Société de Pathologie Exotique. 2015; (108): 324-7.

24. Heller T, Goblirsch S, Wallrauch C, Lessells R, Brunetti E. Abdominal tuberculosis: sonographic diagnosis and treatment response in HIV positive adults in rural South Africa. International Journal of Infectious Diseases. 2010; 14 (Suppl 3): 108-12. DOI: 10.1016/j.jijd.2009.11.030.

\section{References}

1. Gupta P, Kumar S, Sharma V, Mandavdhare H, Dhaka N, Sinha SK et al. Common and uncommon imaging features of abdominal tuberculosis. Journal of Medical Imaging and Radiation Oncology. 2019; 63 (3): 329-39. DOI: 10.1111/1754-9485.12874.

2. Sinitsyn MV, Belilovsky EM, Sokolina IA, Reshetnikov MN, Tityukhina MV, Baturin OV. Extrapulmonary tuberculosis in HIV patients. Tuberculosis and Lung Diseases. 2017; 95 (11): 19-25. DOI: 10.21292/2075-1230-2017-95-11-19-25. Russian.

3. Ködmön C, Zucs P, Van der Werf MJ. Migration-related tuberculosis: Epidemiology and characteristics of tuberculosis cases originating outside the European Union and European Economic Area, 2007 to 2013. Eurosurveillance. 2016; (21): 2327. DOI: 10.2807/1560-7917.ES.2016.21.12.30164.

4. Chow KM, Chow VC, Szeto CC. Indication for peritoneal biopsy in tuberculous peritonitis. The American Journal of Surgery. 2003; (185): 567-73. DOI: 10.1016/s0002-9610(03)00079-5.

5. Plotkin DV, Sinitsyn MV, Reshetnikov MN, Kharitonov SV, Skopin MS, Sokolina IA. Tuberculous peritonitis. «Forgotten» disease. Khirurgiya. Zhurnal im. N.I. Pirogova. 2018; (12): 38-44. DOI: 10.17116/hirurgia20181213. Russian.

6. Vaid U, Kane GC. Tuberculous Peritonitis. Microbiology Spectrum. 2017; (5): 324-29. DOI: 10.1128/microbiolspec.TNMI7-0006-2016.

7. Demirkazik FB, Akhan O, Ozmen MN, Akata D. US and CT findings in the diagnosis of tuberculous peritonitis. Acta Radiologica. 1996; 37 (4): 517-20. DOI: 10.1177/02841851960373P217.

8. Portielje JE, van der Werf SD, Mutsaers JA, Lohle PN, Puylaert JB. Peritonitis tuberculosa echografisch te herkennen [Echographic recognition of tuberculous peritonitis]. Nederlands Tijdschrift voor Geneeskunde. 1997; 141 (2): 89-93.

9. Mojdunova NK, Turdumambetova GK. Ultrasound picture of abdominal tuberculosis. International journal of applied and fundamental research. 2017; (6): 111-3. Russian.

10. Vostrov AN, Mitina LA, Kazakevich VI, Kaprin AD, Stepanov SO, Guts OV, et al. Value of ultrasound in differential diagnosis of ovarian cancer with peritoneal carcinomatosis and peritoneal tuberculosis. Ultrasound and functional diagnostics. 2017; (3): 60-71. Russian.

11. Husain A, Firdaus H, Panday P. Study of Comparison of high resolution sonography and computed tomography in evaluation of abdominal tuberculosis among patients in Lucknow, Uttar Pradesh, India. International Journal of Surgery. 2018; (5): 17139. DOI: 10.18203/2349-2902.isj20181388.

12. Goodman SN. Toward Evidence-Based Medical Statistics. 2: The Bayes Factor. Annals of Internal Medicine. 1999; (130): 1005-13.

13. Smereczyński A, Kołaczyk K, Bernatowicz E. Difficulties in differentiating the nature of ascites based on ultrasound imaging. Journal of Ultrasonography. 2017; 17 (69): 96-100. DOI: 10.15557/JoU.2017.0013.

14. Atzori S, Vidili G, Delitala G. Usefulness of ultrasound in the diagnosis of peritoneal tuberculosis. The Journal of Infection in Developing Countries. 2012; 6 (12): 886-90. DOI: 10.3855/ jidc. 2654

15. Weill FS, Costaz R, Guetarni S, Maltoni I, Rohmer P. Echographic diagnosis of peritoneal metastases in patients with ascites. European Journal of Radiology. 1990; (71): 365-8.

16. Stepanov SO, Mitina LA, Guts OV, Bespalov PD. Ultrasound imaging of peritoneal dissemination. Ultrasound and functional diagnostics. 2013; 3 (4): 66-70. Russian.

17. N'dri K, Gbazi GC, Konan A, Kouadio, Koffi, N'dri N, et al. Apport de l'échographie dans le diagnostic de la tuberculose péritonéale ascitique. Médecine d'Afrique Noire. 1993; (40): 503-6.

18. Dafiri R, Imani F. Tuberculose abdominale. Encycl Méd Chir (Editions Scientifiques et Médicales Elsevier SAS, Paris, tous droits réservés). Radiodiagnostic - Appareil digestif 2001; 33010-A-30: 12

19. Gastli H, Hassine W, Absesselem K, Gharbi HA. Echographic aspects of peritoneal tuberculosis. Apropos of 14 cases. European Journal of Radiology. 1983; (64): 325-9.

20. Mbengue A, Ndiaye AR, Amar N, Diallo M, et al. Ultrasonography of peritoneal tuberculosis. Journal of Ultrasonography. 2019; (19): 98-104. DOI: 10.15557/JoU.2019.0014.

21. Batra A, Gulati MS, Sarma D, Paul SB. Sonographic appearances in abdominal tuberculosis. Journal of Clinical Ultrasound. 2000; (28): 233-45. DOI: 10.1002/(sici)1097-0096(200006)28:5<233::aidjcu5>3.0.co;2-c.

22. Fall F, Ndiaye AR, Ndiaye B, Gning SB, Diop Y, Fall B. Peritoneal tuberculosis: a retrospective study of 61 cases at Principal hospital in Dakar. Journal of Gastroenterology and Hepatology. 2010; (4): 38-43.

23. Darré T, Tchaou M, Sonhaye L, Patassi AA, Kanassoua K, Tchangai B. Analyse d'une série de 44 cas de tuberculose péritonéale diagnostiqués au laboratoire d'anatomie pathologique du $\mathrm{CHU}$ Tokoin de Lomé (1993-2014). Bulletin de la Société de Pathologie Exotique. 2015; (108): 324-7.

24. Heller T, Goblirsch S, Wallrauch C, Lessells R, Brunetti E. Abdominal tuberculosis: sonographic diagnosis and treatment response in HIV positive adults in rural South Africa. International Journal of Infectious Diseases. 2010; 14 (Suppl 3): 108-12. DOI: 10.1016/j.ijid.2009.11.030. 